Cahiers d'études italiennes

\title{
Le differenze culturali e la figura dell'immigrato in Benvenuti in questo ambiente di Carmen Covito
}

Susanne Kleinert

\section{OpenEdition}

\section{Journals}

Edizione digitale

URL: http://journals.openedition.org/cei/930

DOI: $10.4000 /$ cei.930

ISSN: 2260-779X

\section{Editore}

UGA Éditions/Université Grenoble Alpes

\section{Edizione cartacea}

Data di pubblicazione: 15 maggio 2008

Paginazione: $241-251$

ISBN: 978-2-84310-121-2

ISSN: $1770-9571$

Notizia bibliografica digitale

Susanne Kleinert, « Le differenze culturali e la figura dell'immigrato in Benvenuti in questo ambiente di Carmen Covito », Cahiers d'études italiennes [Online], 7| 2008, Messo online il 15 novembre 2009 consultato il 22 avril 2019. URL : http://journals.openedition.org/cei/930 ; DOI : 10.4000/cei.930 


\title{
LE DIFFERENZE CULTURALI E LA FIGURA
}

DELL'IMMIGRATO

\section{IN BENVENUTI IN QUESTO AMBIENTE DI CARMEN COVITO}

\author{
Susanne Kleinert
}

Université de Sarrebruck

\section{Letteratura di migranti e dialogo interculturale}

La letteratura di migranti è - insieme alla letteratura di viaggio - uno dei campi principali degli studi di letteratura interculturale. Se in passato l'Italia ha fornito a questo campo di ricerca soprattutto una letteratura di emigrazion $^{1^{*}}$, la situazione è cambiata con l'arrivo di un numero notevole di migranti in Italia durante gli ultimi decenni, un'immigrazione che comporta anche la nascita di una letteratura di migranti in Italia. L'immigrazione in Italia da paesi del Terzo Mondo è un fenomeno relativamente recente, se paragonato alla Francia. Se in Francia gli studi di letteratura interculturale sono ben radicati, perché già da tempo esiste una letteratura di migranti di varie generazioni, in Italia essi non hanno ancora riscontrato molto interesse, cosa che non stupisce se si pensa alla relativa novità del fenomeno. Inoltre la divisione fra i vari indirizzi di studi letterari non è favorevole alla ricerca sull'interculturalità. In Germania gli scrittori italiani emigrati che hanno scelto di usare il tedesco come lingua letteraria spesso non si sentono presi in considerazione né dalla germanistica né dall'italianistica ${ }^{2}$. Armando Gnisci, studioso di Letteratura comparata e di Letteratura di migranti, rimprovera polemicamente all'Italia di aver praticamente rimosso il suo passato colonialistico, di aver dimenticato la letteratura dei propri emigranti e di essere sorda alle voci dei migranti che oggi contribuiscono a creare una nuova letteratura interculturale 3 .

Come fa notare il critico e scrittore italiano emigrato Carmine Chiellino, il migrante non solo porta la sua cultura d'origine con sé, ma modifica anche la cultura del paese d'arrivo perché crea una diversità all'interno di questa, cosa che tendenzialmente comporta un processo di percezione interculturale, sia dialogico sia conflittuale ${ }^{4}$.

* Notes p. 249. 
In questo senso, la presenza di migranti in Italia si fa notare non solo nella letteratura di migranti ${ }^{5}$, ma anche nella cultura italiana stessa. Nel film Gente di Roma di Ettore Scola (2003), i migranti extracomunitari hanno un ruolo importante perché la loro presenza incita a una riflessione sull'immagine della tolleranza (o indifferenza) romana, quindi sulla capacità dei romani di percepire e tollerare le differenze culturali. Sandro Veronesi, in Gli sfiorati, introduce il suo protagonista Mète in un ambiente di giovani filippini che abitano a Roma e lavorano come domestici. Questi personaggi sono secondari, ma hanno una loro dignità tragica. Nell'epilogo il protagonista è lui stesso emigrato nelle Filippine e parla la loro lingua (non tradotta in italiano nel testo) ${ }^{6}$. Il romanzo si chiude quindi con l'immagine di una diversità culturale adottata dal protagonista italiano.

\section{La figura dell'immigrato in Benvenuti in questo ambiente}

Nel terzo romanzo di Carmen Covito, Benvenuti in questo ambiente (1997), l'immigrato non è più una figura secondaria, bensì il protagonista del romanzo. Avendo passato vari anni in Giappone come moglie di uno studioso giapponese, la Covito è dotata di una particolare sensibilità per le situazioni interculturali. Ha dedicato il suo romanzo a una coppia di nome arabo che definisce come «italiani nuovi». Il romanzo inizia con la descrizione di un dialogo tra un immigrato tunisino di nome Nureddin Djemali e un computer, che funziona anche come riferimento metafinzionale perché si autodefinisce così : «Ho in dotazione un senso dell'umorismo standard. Dispongo anche di : Frivolezza, serietà, sentimento medio e medio-alto ${ }^{7}$. Il dialogo tra Nureddin e il computer struttura l'intero testo, che comprende una serie di flash-backs introdotti dai comandi del programma Word "apri finestra», con riferimento esplicito alla tecnologia informatica. Verso la metà del romanzo Nureddin scopre che Lucia, la madre del proprietario di casa, si nasconde dietro lo schermo del computer. Il primo flash-back racconta l'incontro tra l'immigrato e il proprietario di casa, Ugo Digrosso, un chirurgo estetico di Desenzano.

Quando Nureddin incontra Ugo, che deciderà di assumerlo come custode e cameriere-cuoco tuttofare, ha già alle spalle l'esperienza deludente tipica dell'immigrato. Alla stazione di Bolzano, mezzo morto per il freddo di gennaio, Nureddin ha deciso di tornare verso il Sud, una decisione che equivale a riconoscere il fallimento della ricerca di un posto di lavoro. Nella descrizione dell'incontro fra Nureddin e il suo futuro datore di 
lavoro, Ugo Digrosso, la Covito pone l'accento sul punto di vista di Digrosso e sui cliché da cui è condizionato. Lo stupisce per esempio l'italiano colto di Nureddin, la sua cortesia e il fatto che egli non cerchi di vendergli niente.

Già all'inizio del romanzo Carmen Covito si serve di un doppio registro : all'immagine stereotipata della differenza dell'immigrato rispetto all'autostereotipo dell'italiano (povero e incolto versus benestante e colto) si sostituisce un altro gioco delle differenze : Nureddin è diverso dall'eterostereotipo ${ }^{8}$.

Il contatto fra il ricchissimo Ugo e il povero Nureddin nasce nella situazione fortuita di un treno affollato e viene rafforzato da una situazione di emergenza, perché Nureddin, che non ha mangiato niente da vari giorni, crolla e viene immediatamente soccorso da Digrosso. Quest'ultimo lo assume e lo porta alla sua villa di Desenzano, sul lago di Garda, dove abita con la sorella Sandrina, titolare di una ditta di software. I due italiani non corrispondono al cliché dell'italiano legato in primo luogo alla famiglia. Non hanno figli, si dedicano completamente al lavoro e si lasciano guidare dalle proprie nevrosi ; nel loro atteggiamento, figurano come esempi dello sviluppo economico dell'Italia settentrionale, ma anche di certe tendenze di life style universali. Se Ugo rappresenta l'ossessione del corpo e del suo continuo modellamento, Sandrina vive invece nei mondi dell'intelligenza artificiale ed ha attrezzato la casa di alta tecnologia elettronica. A 23 anni, la ragazza ha una paura nevrotica del declino biologico delle cellule del suo cervello, ma non esita ad aiutare i suoi clienti a licenziare gli ultimi lavoratori ${ }^{9}$. I due fratelli rappresentano ironicamente i settori più avanzati d'Italia, ma il vocabolario anglicizzante di Sandrina dimostra che si tratta solo della parte italiana di un'industria globalizzata. L'autrice commenta la scelta dell'ambientazione del romanzo : "Il romanzo è ambientato nel Nord-Est italiano perché mi è sembrato un luogo di contraddizioni, particolarmente adatto a drammatizzare quelle che sono le attuali contraddizioni nei confronti dell'informatica e della telematica ${ }^{10}$ ».

Dopo la festa di compleanno di Sandrina i due spariscono per vari giorni e Nureddin rimane solo davanti ai tanti computer che apparentemente dirigono anche gli attrezzi di casa. Nureddin scopre che i suoi movimenti vengono controllati dalle videocamere e dal computer - con il quale comincia a comunicare. Egli si sente onorato della fiducia dimostrata dai due fratelli nel lasciarlo lì solo e sente come suo obbligo personale prendersi cura della casa. Questo suo punto di vista viene però contraddetto dal fatto che Sandrina gli prende il passaporto, trattandolo 
quindi come una persona non degna di fiducia e per di più dipendente dalla volontà del padrone di casa. Le due prospettive implicano un'asimmetria. Se, dal canto loro, i due ricchi italiani percepiscono Nureddin solo in virtù della funzione assegnatagli in casa, lui, dal canto suo, vorrebbe invece essere stimato come persona.

Il romanzo comincia quindi con la creazione di un forte contrasto fra Nureddin e il lussuoso ambiente italiano. L'atteggiamento degli italiani rispetto a Nureddin non è caratterizzato da un aperto razzismo. I due fratelli sono piuttosto indifferenti e talmente presi dai propri problemi personali e professionali che non si curano della presenza di Nureddin. Dall'altro lato la figura di Nureddin non rappresenta tanto il lato sociale della questione dell'immigrazione, quanto piuttosto l'aspetto psichico e identitario. Egli non conferma il cliché dell'immigrato povero e incolto, perché è cresciuto all'Hotel Hilton di Tunisi e sa muoversi in un ambiente internazionale. Il suo personaggio riveste nel romanzo una funzione particolare : la descrizione dell'ambiente di lusso attraverso la sua percezione assume una valenza caricaturale. A causa del suo sentimento della dignità personale da un lato, e delle varie nevrosi dei due fratelli e dei loro amici dall'altro, sorge una serie di malintesi che conferiscono al romanzo una comicità interculturale. I complessi d'inferiorità di Nureddin sono condizionati dalle norme della sua società di origine, ma sono superflui nella società italiana, cosa che lui però non sa. Al pudore di Nureddin si contrappongono le invadenti strategie di seduzione di Annapaola, una grande consumatrice di uomini (BIQA, p. 100-105), o la transessualità di un altro personaggio operato da Digrosso (p. 54). In questo contrasto i due atteggiamenti vengono ironizzati dall'autrice. Per quanto riguarda il tema della sessualità e del corpo, la Covito si serve del grottesco per sottolineare il contrasto fra le società nordafricane e gli atteggiamenti molto liberi dei giovani italiani, descritti come narcisisti e sfruttatori. Si potrebbe dire che nel romanzo della Covito il giovane tunisino soffre dei suoi complessi sessuali e i giovani italiani invece di una mancanza di empatia sentimentale. Ma questa critica implicita delle due culture non viene drammatizzata, bensì trasmessa in modo più leggero attraverso un registro comico-grottesco.

La Covito si è molto preoccupata di non rinchiudere la figura dell'immigrato in una serie di clichés. Nureddin non è un rappresentante tipico della società tunisina, ma raffigura piuttosto il migrante, cioè una persona che si trova in una posizione intermedia fra due culture. Con la creazione di questo personaggio l'autrice si è allontanata decisamente dal carattere di letteratura documentaria molto diffuso nella letteratura della 
migrazione. La biografia di Nureddin sottolinea il suo ruolo di mediazione : egli è infatti il frutto di un breve amore tra un cuoco tunisino dell'Hotel Hilton di Tunisi e una hippy italiana, che lascia il bambino a suo padre perché vuole realizzarsi andando in India come Sanyassin del Bhagwan Shree Rajneesh. Nella scena dell'incontro durante il quale la madre lascia Nureddin al cuoco Yussef, l'autrice gioca ironicamente sulle differenze di percezione di entrambe le culture. Auto- ed eterostereotipo formano un contrasto divertente : mentre la madre di Nureddin è estasiata di fronte ai tramonti sul deserto e sulla città di Tunisi e immagina per il figlio una vita " autentica ${ }^{11}$ ", il padre ha ben altri progetti :

[...] mai e poi mai avrebbe consegnato la produzione delle proprie viscere a un branco di ignoranti tunisini, donne di casa o maestri di scuola che fossero : suo figlio, nato internazionale, era predestinato a diventare un europeo famoso, un Paul Bocuse, un Joël Robuchon, o, per considerare la nazionalità dell'utero dal quale gli era stato scodellato, perlomeno un Gualtiero Marchesi. (BIQA, p. 72)

L'autrice non rappresenta le differenze culturali in modo essenzialistico, ma come proiezioni di eterostereotipi : mentre la madre hippy di Nureddin cerca la vita "autentica» fuori dall'Europa, secondo lo spirito degli anni 1970, Yussef coltiva invece il sogno di un'Europa raffinata, che consente la liberazione dalle costrizioni della società tunisina. Per questo motivo Yussef fa crescere Nureddin come un clandestino lontano dalla società tunisina, in quel luogo di passaggio tra le culture dove lui stesso lavora, l'Hotel Hilton di Tunisi. Il testo sottolinea in questo modo che dà scarso valore alla questione dell'origine ${ }^{12}$.

La Covito non ha creato la figura dello straniero simbolo di autenticità e naturalezza in opposizione alla decadenza della cultura occidentale. Nureddin non possiede un'identità stabile. Uno dei motivi per cui è andato in Italia è la ricerca della madre e delle ragioni del suo abbandono. Essendo privo di madre e straniato anche dalla società tunisina per volontà del padre, non si sente legato alla famiglia o ad un collettivo sociale. Il suo soprannome "Light» sottolinea proprio questo fatto : il doppio significato di "Light» (luce e leggero) rimanda alla sua fragile identità $(B I Q A$, p. 17). Il padre gli ha trasmesso la cultura occidentale, ma non gli ha insegnato l'arabo. Nureddin ha quindi interiorizzato un'immagine molto positiva della cultura italiana, ed europea in genere, alla quale però gli stessi italiani non credono più. Mentre Nurreddin ha letto molti libri europei all'Hotel Hilton di Tunisi e possiede una discreta cultura letteraria, Ugo Digrosso legge solo studi di chirurgia e fumetti - un dettaglio significativo che sottolinea la differenza fra i nuovi ricchi italiani e l'immagine che Nureddin ha dell'Europa (BIQA, p. 83-84). 
L'illusione del padre di Nureddin di potergli garantire uno splendido futuro in Europa solo tramite la padronanza delle lingue e della cultura europea viene smentita dall'esistenza reale del figlio, che non ha nemmeno un passaporto tunisino - "come mai nato, come mai vissuto" (BIQA, p. 107) - e, dopo la morte del padre, decide di andare in Italia come immigrato clandestino. Il carattere interculturale della figura di Nureddin è anche evidente in una scena di sogno : egli immagina di trovarsi in una casa occidentale di sua proprietà che però si restringe sempre di più, e nella quale si muove non come un corpo bensì come « una forma fatta di pura luce» (BIQA, p.117). Dato che Nureddin non appartiene ad una determinata cultura, la sua condizione e la sua identità sono leggere. Com'è evidente dal sogno citato, egli vive la propria situazione con un senso di angoscia. Alla fine del romanzo la Covito comunica però una visione piuttosto positiva di questa condizione. Nureddin viene esplicitamente definito una figura che rappresenta la postmodernità :

Senza parole - non possiede ancora parole sufficienti per un pensiero tanto acrobaticamente solipsista, tanto assolutamente postmoderno - Nureddin si sta vedendo andare per la vita con se stesso come tutto bagaglio, e la cosa gli piace. Non ha origine, lui. Lui è puro uomo. Generato in un transito casuale, sgusciato tra due mondi approfittando di un interstizio... (BIQA, p. 246)

La figura di Nureddin rappresenta quindi una postmodernità che evita i discorsi identitari, come sottolinea la costante ironia del romanzo. Anche la fine aperta corrisponde a questa linea del romanzo, che non si chiude con la classica scena del ritrovamento della madre di Nureddin : è una fine che lascia aperti i conflitti e che non conclude. Ė più importante l'autodefinizione di Nureddin, che ha il coraggio di definirsi «normale» ad alta voce (BIQA, p. 240). Analogamente ai due primi romanzi dell'autrice ${ }^{13}$, anche questo romanzo contiene tracce del romanzo di formazione : Nureddin impara a liberarsi da un'immagine dell'identità che si basi unicamente sulla questione dell'origine e comincia ad apprezzare la propria posizione a cavallo fra due culture. Questa evoluzione interna viene rappresentata simbolicamente dal fatto che egli riesce ad abbandonare la ricerca della madre italiana, innamorandosi della Dama del Computer. Non impara solo a muoversi nello strano ambiente dei nuovi ricchi di Desenzano, ma anche a riflettere su se stesso in un altro modo, accettando la propria identità leggera, tipicamente postmoderna. 


\section{Interculturalità/transculturalità e voce narrativa}

Che rapporto ha questa immagine della condizione «light» dell'immigrato con la rappresentazione della società di origine di Nureddin? Anche se la Covito non approfondisce molto il tema delle radici nordafricane di Nureddin, spiega l'origine del suo sentimento di sradicamento inserendo nel romanzo un brano su Tunisi. Nella scena del matrimonio dello zio, che forse è il suo padre biologico, Nureddin scopre una società molto gerarchica e rappresa in tradizioni assurde che costringono gli individui a mentire e dissimulare. Inoltre i suoi coetanei tunisini trattano Nureddin in modo violento ed umiliante quando scoprono che lui non è circonciso e perciò lo identificano come «cristiano" (BIQA, p. 176-192). Escluso in questo modo dalla propria famiglia, Nureddin mette a fuoco la società nordafricana in modo abbastanza critico. Ma dal punto di vista di Nureddin anche gli interessi e i comportamenti degli italiani appaiono strani e il lettore condivide facilmente il suo giudizio ("Che famiglia di matti», BIQA, p. 57), quando osserva il consumismo e i litigi sfrenati dei due fratelli e l'atteggiamento bizzarro dei loro amici. Visti dalla prospettiva di Nureddin, i tentativi di Annapaola di sedurre il giovane tunisino appaiono insensibili, aggressivi e irrispettosi delle differenze culturali (BIQA, p. 100-105). Altri amici, come Marco, il fidanzato di Sandrina, si perdono in Internet e nei mondi della fantascienza e dell'orrore ${ }^{14}$. La figura dell'immigrato assume quindi la funzione di specchio critico e straniante rispetto alle due culture, sia la tunisina che l'italiana.

La voce narrativa è strutturata in modo complesso. L'azione viene raccontata nei file del computer da una voce misteriosa che verso la metà del libro si scopre essere quella di Lucia, la madre dei due fratelli. Nelle varie "finestre» viene intercalato il dialogo diretto fra Nureddin e la Dama del Computer. A metà del romanzo si trova anche una riflessione metafinzionale presentata come un Easter Egg nel gergo dei programmatori ${ }^{15}$. La narratrice dei file oscilla fra focalizzazione esterna ed interna degli altri personaggi. Carmen Covito commenta la struttura narrativa nel modo seguente :

Insomma, io credo che la narrativa tradizionale non debba più accontentarsi delle strutture letterarie classiche, ma debba in ogni modo far entrare nei suoi canoni quelle che sono le modificazioni della nostra percezione. E la nostra percezione oggi è modellata innanzitutto dai mass media (primo in ordine cronologico la televisione), ma ora anche dalle interfacce dei computer ${ }^{16}$.

Quanto all'azione, la Dama del Computer ha una funzione ambigua nel romanzo. Da un lato fa scoprire a Nureddin la sua «deviazione» - cioè 
il fatto di non essere circonciso - come normale, portandolo così ad accettare la propria virilità, dall'altro lato è un personaggio straniato e mostruoso per la sua deformità fisica ${ }^{17}$. Il personaggio implica anche una funzione di commento psicologico, ma d'altra parte la sua violenza può indurre il lettore a seguire il giudizio di Sandrina, che considera la madre una pazza da rinchiudere definitivamente in cantina. Dato che la Dama del Computer scrive i file che costituiscono il racconto, la voce narrativa stessa acquisisce una certa ambiguità.

Già all'inizio del romanzo Ugo paragona il soprannome di Nureddin, Light, al nome Lucia di sua madre ( $B I Q A$, p. 17). L'avvicinamento fra la narratrice e il migrante struttura il racconto, entrambi hanno una funzione straniante ma vengono a loro volta straniati attraverso la costante ironia del romanzo. Alla fine l'avvicinamento fra i due personaggi si sposta dal livello sentimentale a quello elettronico : Sandrina allestisce una pagina Internet per sua madre e Nureddin, sulla quale lui pubblicherà le sue ricette di alta gastronomia multietnica. La stessa struttura narrativa evita quindi di costruire un'istanza centrale da cui vengano emessi i commenti sui personaggi. La focalizzazione flessibile acquisisce un valore culturale perché viene associata al dialogo fra la narratrice e il migrante e alla tematica di confronto interculturale.

Il riferimento a Internet s'inserisce in una serie di rimandi alla cultura mediatica contemporanea. Sia Nureddin che i suoi coetanei italiani cercano di capire la realtà orientandosi secondo modelli presi dal cinema, dalla televisione o da Internet ${ }^{18}$. Con i suoi rinvii ad una cultura mediatica comune l'autrice trascende il livello di paragone interculturale, cioè di giustapposizione di due culture distinte, una giustapposizione che è tuttavia presente nel romanzo nei brani che mettono a confronto la cultura nordafricana con i settori economicamente e tecnologicamente avanzati della cultura occidentale. Nel dibattito teorico sull'interculturalità, si distingue fra interculturalità (nel senso di dialogo o conflitto fra due culture diverse) e transculturalità (nel senso di una pluralità d'identità culturali che non permette più di fissare un limite preciso fra due culture ${ }^{19}$ ). Con $\mathrm{i}$ grandi processi di migrazione e di globalizzazione dell'economia, le esperienze transculturali aumentano, e Carmen Covito sembra esserne consapevole. Il modo in cui il romanzo si serve di allusioni al cinema e ai nuovi media fa capire al lettore che i media creano dei punti di convergenza fra le varie culture. Covito vede i processi transculturali in un'ottica positiva e si dichiara attratta dalle implicazioni sociologiche, psicologiche e filosofiche di Internet, ossia «l'azzeramento delle distanze e dei confini geografici attraverso la simultaneità della comunicazione, la frantumazione e la 
moltiplicazione dell'io ${ }^{20} »$. In primo luogo difende i diritti dell'individuo contro i concetti d'identità collettiva, come dimostra nel romanzo l'episodio dell'aggressione dei giovani tunisini contro Nureddin. Inoltre, in un'intervista sui personaggi e il punto di vista nel romanzo, sostiene che grazie ai media il mondo di oggi è diventato "più imprendibile, ma molto più vicino ${ }^{21}$ ». Ciò che dice sulla condizione moderna/postmoderna in questa intervista è applicabile anche alla questione del come vivere in un mondo multiculturale e policentrico :

Dobbiamo accettare serenamente questa nozione : non abbiamo centro e direi addirittura che l'essenza della modernità consiste nel non sapere che farsene di un centro. Questa consapevolezza può fare anche molta paura...

Ma no !!! Magari perdessimo tutti i centri che ancora resistono. La nostra sfida di moderni o, se preferisce, di postmoderni, è questa : camminare sul vuoto. E bisogna affrontarla con una certa baldanzosità ${ }^{22}$.

Il tema delle differenze culturali affrontato dall'autrice nel suo terzo romanzo riflette quindi una presa di posizione più ampia dei soli aspetti tematici. Nel caso della Covito si può parlare di una estetica della differenza, di una visione frammentaria della realtà, come dimostra un altro brano della stessa intervista : "Il romanzo può ancora avere una funzione conoscitiva, può dirci qualcosa di sconvolgente, di imprevisto, di inedito, di totale : però deve farlo, ormai, attraverso una simulazione frammentaria della realtà̀ 23 .

In sintesi, in Benvenuti in questo ambiente la figura del migrante assume la funzione di rispecchiamento straniante di tendenze attuali della nostra società e rappresenta il dialogo interculturale nelle sue difficoltà ma anche nel lato comico-grottesco dei suoi malintesi. Inoltre, nel suo terzo romanzo l'autrice presenta la sua visione di una cultura policentrica, aperta alle differenze culturali, ma anche lontana dall'idealizzare condizioni culturali ostili alle scelte personali degli individui. Sottolineando l'influenza dei media sulla percezione, l'autrice dimostra anche di essere consapevole dei punti di contatto transculturali inerenti al processo di globalizzazione.

\section{Notes}

1. Cfr. La letteratura dell'emigrazione. Gli scrittori di lingua italiana nel mondo, a. c. di J.-J. Marchand, Torino, Edizioni della Fondazione Giovanni Agnelli, 1991. Per la letteratura di emigrati italiani in Germania, vedi le opere di C. Chiellino, p. es. «Parole erranti. Emigrazione, letteratura e interculturalità", in Saggi 1995-2000, (Quaderni sull'emigrazione 5), Isernia, Cosmo Iannone Editore, 2001 ; e Am Ufer der Fremde. Literatur und Arbeitsmigration 1870-1991, Stoccarda, Metzler, 1995. 


\section{SUSANNE KLEINERT}

2. Lo studioso della letteratura italo-tedesca di migrazione Carmine Chiellino è lui stesso poeta e migrante. In Parole erranti lamenta una mancanza di dialogo fra intellettuali tedeschi ed intellettuali migranti (p. 89) e della scarsità di strumenti critici a disposizione dell'italianistica italiana per studiare gli autori migranti (p.95).

3. Vedi A. Gnisci, Creolizzare l'Europa, Roma, Meltemi, 2003, p. 145-146.

4. Vedi C. Chiellino, "Parole erranti», p. es. il capitolo sulla memoria biculturale, p. 77-85.

5. p. es. M. Fortunato, S. Methnani, Immigrato, Roma-Napoli, Theoria, 1990 e 1997.

6. S. Veronesi, Gli sfiorati, Milano, Mondadori, 1990 e 2001. Vedi anche gli altri contributi in questo volume dedicati al tema delle differenze interculturali.

7. C. Covito, Benvenuti in questo ambiente, Milano, Bompiani, 1997, p. 5 , in seguito abbreviato $B I Q A$.

8. In questo senso Nureddin si può paragonare a M. Fortunato e S. Methnani, Immigrato : anche il narratore di questo testo documentario fa mostra delle proprie conoscenze culturali. Non è un immigrato incolto, bensì laureato e dotato di una cultura cinematografica che comprende perfino i film di Fassbinder (cfr. p. 12 e 116). Per il concetto di auto- ed eterostereotipo vedi il saggio ormai classico sulla comunicazione interculturale : G. Hofstede, Cultures and organizations : Software of the Mind, London, McGraw-Hill Book Company, 1991, p. 212.

9. L'immagine delle ditte italiane è ironica, vedi p. es. la seguente riflessione di Sandrina su come trattare un cliente : "Tordelli Posaterie di Lumezzane, sta rognando perché il Controller Catena che gli abbiamo fornito concede un intervallo-pipì ai lavoratori. Obiettargli che il nostro sistema gli ha già permesso di ridurre la manodopera umana a soli tre operai senegalesi. Intimidirlo ricordandogli che cinque minuti su otto ore sono il minimo per non incappare nella Convenzione di Ginevra sui prigionieri di guerra. Se non ride, proporgli di upgradare il sistema passando alla versione 5.1, per fabbrichette totalmente robotizzate.» (BIQA, p. 38). La scena del compleanno di Sandrina (p.37-59) introduce il lettore nell'ambiente sofisticato dei giovani professionisti dell'Italia settentrionale.

10. R. Capozzi, "Un incontro elettronico con Carmen Covito", in Forum Italicum 33, 1, Spring 1999, p. 271. $71)$.

11. "Ma è un incanto, un incanto! Mio figlio crescerà qui, tra i suoi antenati ..." (BIQA, p.

12. Infatti, il testo fornisce tre versioni della relazione fra Yussef e la madre di Nureddin (p. 6779) ; la terza suggerisce che il padre biologico di Nureddin non è Yussef, ma lo zio Hassan, il portiere di notte dell'Hilton. Vedi anche il consiglio dato a Nureddin dalla Dama del computer per dissuaderlo dal progetto di trovare sua madre in Italia : «Lascia stare, Nureddin. Certe madri è meglio perderle che trovarle." (BIQA, p. 150).

13. Sulle opere della Covito vedi S. Kleinert, "Carmen Covitos Italienbilder : Geschichten von narzisstischen, elektronischen und interkulturellen Beziehungen", in Italienische Erzählliteratur der Achtziger und Neunziger Jahre, a. c. di F. Balletta e A. Barwig, Francoforte et al., Peter Lang, 2002.

14. Marco, come Sabrina, tende a perdersi in un mondo virtuale, perché "vede tutto il mondo un po' sfasato, come attraverso un filtro polaroid sporchiccio che gli mostra gli oggetti e le figure tutti clonati di virtualità.» $(B I Q A$, p. 161). Un altro personaggio, Carol Invernizio, rimanda ironicamente ai romanzi popolari di Carolina Invernizio.

15. In questa sequenza, scritta in corsivo, il narratore si autodefinisce così : «Abbastanza onnisciente ma divinamente discreto, sono un occhio orientabile, un orecchio direzionale, una macchina celibe in agguato per processare un po' di vite altrui. Non le giudico, al massimo le salvo : configuro, scandisco, seleziono, sincronizzo, ottimizzo, interconnetto, codifico, formatto e immagazzino una campionatura della vita ; poi, tramite tastiera, la riverso su carta.» (BIQA, p. 115)

16. R. Capozzi, op. cit., p. 269.

17. Lucia spiega a Nureddin che in Europa è normale per un uomo non essere circonciso, cosa che lui non sapeva, essendo cresciuto in una situazione d'isolamento dai coetanei (p. 175-176). Spiega anche perché si è messa a disposizione di Ugo come oggetto di esperimenti chirurgici e come si è trasformata in una specie di Artemide di Efeso lasciandosi applicare otto seni artificiali in aggiunta ai due naturali. Il racconto di Lucia sulle sue operazioni di chirurgia estetica è caricaturale, 
ma ha anche un lato ossessivo, di modo che il lettore non può fidarsi completamente dello stato mentale del personaggio (vedi anche l'autoritratto di Lucia come donna probabilmente psicotica, p. 132-146). Il lato ossessivo del personaggio diventa evidente nel suo gesto di ferire Nureddin con una forchetta dopo il coito, un gesto che però acquisisce anche una funzione pedagogica : lo vuole distanziare da sé perché sa di essersi offerta a Nureddin come un sostituto della madre.

18. Cfr. il ruolo dei film western nell'immaginazione di Nureddin (BIQA, p. 179) e i riferimenti ai vari James Bond (p. 153), al film Blade Runner (p. 164), alle serie televisive, p. es. X-Files (p.159), soprattutto nell'immaginazione di Marco.

19. H.-J. Lüsebrink, Interkulturelle Kommunikation, Stuttgart, Weimar, Metzler-Verlag, 2005, p. 17-18.

20. R. Capozzi, op. cit., p. 268.

21. L. Lepri, «Intervista a Carmen Covito, I personaggi e il punto di vista», in Scrittura creativa. La scrittura creativa raccontata dagli scrittori che la insegnano, Milano, Bompiani, 1998, p. 103.

22. Ibidem, p. 101.

23. Ibidem, p. 102. 